\title{
Radium-223 in a Community Setting for Castration Resistant Prostate Cancer
}

\author{
Houman Vaghefi ${ }^{1}$, Sachin Agarwal ${ }^{2}$, Fang Liu $^{3}$, Bide Xiong ${ }^{4}$, Elizabeth Garber ${ }^{5} \&$ James Wheeler $^{6}$ \\ ${ }^{1}$ Attending Physician, Department of Radiation Oncology, Goshen Center for Cancer Care, United States \\ ${ }^{2}$ Attending Physician, Department of Medical Oncology, Goshen Center for Cancer Care, United States \\ ${ }^{3}$ Assistant Professor, Department of Applied and Computational Mathematics and Statistics, University of Notre \\ Dame, United States \\ ${ }^{4}$ Graduate Student, Department of Applied and Computational Mathematics and Statistics, University of Notre \\ Dame, United States \\ ${ }^{5}$ Nurse Practitioner, Department of Radiation Oncology, Goshen Center for Cancer Care, United States \\ ${ }^{6}$ Attending Physician and Medical Director, Department of Radiation Oncology, Goshen Center for Cancer Care, \\ United States \\ Correspondence: James Wheeler, Medical Director, Department of Radiation Oncology, Goshen Center for \\ Cancer Care, United States. E-mail: jwheeler@goshenhealth.com
}

Received: December 13, 2016 Accepted: November 12, $2016 \quad$ Online Published: January 18, 2017

doi:10.5539/cco.v6n1p44 URL: http://dx.doi.org/10.5539/cco.v6n1p44

\begin{abstract}
Purpose: To evaluate the toxicity, palliative benefit, and survival benefit of Radium-223 (Ra-223) in a community setting.

Introduction: The ALSYMCA (Alpharadin in Symptomatic Prostate Cancer) trial demonstrated improved survival for men with painful bone metastasis from castration resistant prostate cancer who were treated with Ra-223. The median survival was 14.9 months for the Ra-223 patients compared to 11.3 months for patients treated with a placebo.
\end{abstract}

Methods: We identified through the nuclear medicine records 11 patients who started Ra-223 between March 15, 2013 and December 10, 2015. The Wilcoxon signed rank test was used to compare the published results of the ALSYMPCA patients with those from our institution.

Results: The Hodges-Lehman estimate of the median survival of our patients from the date of the first Ra-223 infusion was 7.8 months. The $95 \%$ confidence interval (CI) was $(2.8,14.7 ; \mathrm{p}=0.0122)$ indicating our patients had a significantly shorter survival than the Ra-223 ALSYMPCA patients.

Our patients did not have a statistically significant worse rate of anemia, neutropenia, or thrombocytopenia.

We used 3 measures of treatment effectiveness: 1) reduction in the pain scores, 2) reduction in the serum PSA, and 3) reduction in the alkaline phosphatase from baseline, but the reductions were not statistically significant.

Conclusions: This small study is not meant to challenge the results of the ALSYMPCA international trial. It is intended only as a caution that prior lines of therapy, especially with newer agents such as abiraterone and enzalutamide, may attenuate the benefit from this treatment, particularly in elderly patients.

Keywords: Radium-223, castrate resistant prostate cancer, bone metastasis

\section{Introduction}

Prostate cancer can exhibit a broad clinical spectrum, from an indolent lesion appropriately managed with observation, to a widely metastatic, castrate resistant, lethal malignancy. Approximately $3 \%$ of patients with newly diagnosed prostate cancer present with bone metastasis, and another $12 \%$ develop bone metastasis within a few years (Norgaard et al., 2010). More than $80 \%$ of prostate cancer patients have metastasis only to the bone (Hess et al., 2006). Risk factors for the subsequent development of bone metastasis include higher Gleason score, higher serum prostate specific antigen (PSA), and shorter PSA doubling time (Moriera et al., 2016). Roughly $40 \%$ of men with metastatic prostate cancer develop skeletal related events (SREs) such as a pathologic fracture, 
spinal cord compression, and the need for surgery or radiotherapy to the involved bone (McDougall et al., 2016). These SREs carry with them substantial economic costs (Perrault et al., 2015), patient discomfort, and reduced cancer specific survival (Koo et al., 2015).

Radium-223 dichloride was developed as a potential palliative treatment for patients with symptomatic bone-only metastasis. The ALSYMCA (Alpharadin in Symptomatic Prostate Cancer) trial was a phase 3, placebo controlled trial that randomized 921 men with bone metastasis from hormone refractory prostate cancer (HRPC) to receive Radium-223 (Ra-223) or placebo roughly every four weeks for a total of six cycles (Parker et al., 2013). Patients had to have at least two bone metastases, have symptoms requiring regular use of narcotic medications, or to have undergone palliative external beam radiation therapy within the 12 weeks prior to trial registration. They were excluded if they had evidence of visceral metastasis or any lymph node greater than $3 \mathrm{~cm}$ in short axis diameter. Ra-223 produced a 30\% survival benefit, with a median survival of 14.9 months compared to 11.3 months for patients treated with a placebo, which led to FDA approval in 2013.

A subsequent pre-specified subgroup analysis evaluated the efficacy and toxicity of Ra-223 for those patients who previously were treated with docetaxel compared to those who did not receive it, and the survival benefit was comparable (Hoskin et al., 2014). The median survival of the docetaxel patients treated with Ra-223 was 14.4 months, $95 \%$ CI $(12.5,15.5$ months) compared to the docetaxel placebo patients who had a median survival of 11.3 months, 95\% CI (10.0,12.9 months). The Ra-223 patients not previously treated with docetaxel had a median survival of 16.1 months, 95\% CI (13.9, 17.8 months) while the placebo patients not previously treated with docetaxel had a median survival of 11.5 months, $95 \%$ CI $(9.5,14.1$ months).

We sought to compare how our patients who received Ra-223 fared compared to the ALSYMPCA patients, particularly since several of our patients were also treated with some of the more recently approved anti-hormonal agents either before or following Ra-223.

\section{Methods and Materials}

The study institution is an approximately 100 bed community hospital based cancer center located in a rural community of 32,000. The hospital Institutional Review Board approved our performing this retrospective analysis. We identified through the nuclear medicine records 11 patients who started Ra-223 between March 15, 2013 and December 10, 2015. Three of these patients received Ra-223 on an expanded access program prior to FDA approval of the radiopharmaceutical. None of our patients were enrolled in the ALSYMPCA trial.

The Wilcoxon signed rank test was used to compare the published results of the ALSYMPCA patients with those from our institution. The Hodges-Lehman estimate of the median survival of our patients was calculated from the date of the first Ra-223 infusion. P-values from all hypothesis tests were compared with the significance level of 0.05 to determine statistical significance.

\section{Results}

Table 1 compares the patient characteristics of the patients on the ALSYMPCA trial who were randomly assigned to receive Ra-223 prior to their first infusion with the patients from our institution. The most striking difference is in patient age, with our patients having a median age of 81 compared to 71 for the ALSYMPCA patients. $73 \%$ of our patients were over 75 years of age, compared to $28 \%$ of the ALSYMPCA patients.

\subsection{Survival}

The ALSYMPCA trial demonstrated a 30\% survival benefit for the patients who received Ra-223, with a median survival of 14.9 months with Ra-223 compared to 11.3 months for patients receiving placebo. The Hodges-Lehman estimate of the median survival of our patients from the date of the first Ra-223 infusion was 7.8 months with a $95 \%$ confidence interval $(\mathrm{CI})$ of $(2.8,14.7)$. The p-value was 0.0122 from testing whether the median survival in our setting was different from 14.9 months, indicating our patients had a statistically significantly shorter median survival than the estimated median survival time from the ALSYMCA trial. The median survival for our patients from the last Ra-223 was 3.53 months.

When comparing the survival from the last Ra-223 infusion for our three patients who completed all six infusions to the eight who received fewer than six Ra-223 infusions, the median survival time difference was 52 days, with a 95\% CI $(-546,263)$, which was not statistically different (p-value >0.999). Similarly, when comparing the survival time from the first Ra-223 infusion for our three patients completing all six infusions to the eight who received fewer infusions, the median survival time difference was 111 days with a 95\% CI (-497, 323), which was not statistically significant ( $p$-value $=0.667$ ). 
Table 1. Baseline comparison of the ALSYMPCA patients who received Radium-223 dichloride compared to institution patients

\begin{tabular}{lll}
\hline & ALSYMPCA & INSTITUTION \\
\hline Number of patients & 614 & 11 \\
Patient age median (range) & $71(49-90)$ & $81(65-89)$ \\
Age >75: \# patients (\%) & $171(28)$ & $8(73)$ \\
ECOG $\leq 1$ : \# patients (\%) & $536(87)$ & $11(100)$ \\
Hemoglobin (g/dL) median (range) & $12.2(8.5-15.7)$ & $12.7(9.8-14.0)$ \\
Alk Phos: Median (range) & $211(32-6431)$ & $120(57-1242)$ \\
Alk Phos <220 (\# patients, \%) & $348(57)$ & $9(82)$ \\
Alk Phos >220 (\# patients, \%) & $266(43)$ & $2(18)$ \\
Current use of bisphosphonates & $250(41)$ & $2(18)$ \\
EBRT within 12 weeks (\#, \%) & $99(16)$ & $3(27)$ \\
Number of bone metastasis & $\#$ patients $(\%)$ & $\#$ patients $(\%)$ \\
Less than 6 & $100(16)$ & $2(18)$ \\
6-20 & $262(43)$ & $3(27)$ \\
$>$ 20 & $195(32)$ & $6(54)$ \\
Superscan & $54(9)$ & $0(0)$ \\
\hline
\end{tabular}

Alk phos: Alkaline phosphatase U/L

EBRT: External beam radiation therapy

\subsection{Tolerance}

Only $27.3 \%$ (95\% CI: $6.0,61.0 \%$ ) of our patients completed all six planned infusions, statistically significantly less than $63 \%$ for the ALSYMPCA patients treated with Ra-223 ( $\mathrm{p}=0.0239)$, but not statistically different than the $47 \%$ completion rate of the ALSYMPCA placebo patients ( $\mathrm{p}=0.236$ ), who completed a median number of five infusions. The median number of infusions for our patients was four.

Only one of our eight patients who stopped Ra-223 prior to the completion of six infusions did so specifically due to side effects. This patient stopped treatment after two infusions due to persistent anemia. Five of our eight patients stopped Ra-223 early due to progressive malignancy or clinical decline, with two going directly into hospice. One patient stopped Ra-223 to try one of the newer agents. There was no documented reason for one patient's decision to stop Ra-223 early.

\subsection{Anemia}

$10 \%$ of the ALSYMPCA patients developed grade 3 or higher anemia, compared to $18 \%$ of our patients. The $95 \%$ confidence interval $(\mathrm{CI})$ was $(2.28,52.78) \%$. The p-value was 0.303 , suggesting the proportion of patients with grade 3 or higher anemia following the Ra-223 treatment in our community hospital based cancer center was not statistically significantly different from $10 \%$.

\subsection{Neutropenia}

$7 \%$ of the ALSYMPCA patients developed grade 3 or higher neutropenia, compared to $9 \%$ of our patients, with a $95 \% \mathrm{CI}$ of $(0.23,41.28) \%$. The p-value was 0.550 , suggesting the proportion of patients with grade 3 or higher neutropenia following the Ra-223 treatment in our cancer center was not statistically significantly different from $7 \%$.

\subsection{Thrombocytopenia}

$7 \%$ of the ALSYMPCA patients developed grade 3 or higher thrombocytopenia, compared to $9 \%$ of our patients, with a $95 \% \mathrm{CI}$ of $(0.23,41.28) \%$. The p-value was 0.550 , suggesting the proportion of patients with grade 3 or higher thrombocytopenia following the Ra-223 treatment in our center was not statistically significantly different from $7 \%$.

We used three measures of treatment effectiveness: 1) reduction in the pain scores, 2) reduction in the serum PSA, and 3) reduction in the alkaline phosphatase. These results are displayed in Table 2. Compared to the pre-treatment values, numerical reductions were seen at the last follow-up evaluation for both the pain scores and the alkaline phosphatase levels, but the reductions were not statistically significant. 
Table 2. Comparison of measurements at the time of the last Radium-223 dichloride infusion and at the time of last follow up compared to the day of the first Radium-223 dichloride infusion.

\begin{tabular}{lll}
\hline Measurement & Hodges-Lehman estimate of median change & p-value from testing \\
\hline & (last vs. pre) & median change $<0$ \\
\hline & (Date of last Ra-223 infusion versus pre Ra-223) & \\
Pain score & 0.023 & 0.572 \\
Alkaline Phosphatase(U/L) & -23 & 0.172 \\
Serum PSA (ng/mL) & 17.3 & 0.951 \\
& (Date of last follow up versus pre Ra-223) & \\
Pain score & -2 & 0.291 \\
$\begin{array}{l}\text { Alkaline } \\
\text { (U/L) }\end{array}$ & -20 & 0.634 \\
\hline
\end{tabular}

Ra-223: Radium-223 dichloride

Pre-Ra-223: Last measurement obtained prior to or the day of the first Radium-223 dichloride infusion

Our patients received several newer agents that were not available to the patients on the ALSYMPCA trial. Table 3 lists these agents (except for docetaxel, which was available at the time of the ALSYMPCA trial) and the number of our patients who received them either before or after Ra-223. In addition, three patients received palliative radiotherapy before their first Ra-223 infusion and three patients received palliative radiotherapy upon completion of Ra-223. Table 4 lists the number of patients who received various numbers of these agents prior to starting Ra-223.

Table 3. Number of patients receiving a drug before/after Radium-223 dichloride

\begin{tabular}{lll}
\hline Drug & Before Ra-223 & After Ra-223 \\
\hline Abiraterone & 5 & 3 \\
Denosumab & 3 & 0 \\
Docetaxel & 2 & 3 \\
Enzalutamide & 1 & 1 \\
\hline
\end{tabular}

Table 4. Number of patients receiving a given number of drugs before/after Radium-223 dichloride

Ra-223: Radium-223 dichloride

\begin{tabular}{lll}
\hline Number of Drugs & Before Ra-223 & After Ra-223 \\
\hline 0 & 4 & 6 \\
1 & 2 & 4 \\
2 & 5 & 0 \\
3 & 0 & 1 \\
4 & 0 & 0 \\
\hline
\end{tabular}

\section{Discussion}

Radium-223 dichloride selectively binds to areas of increased bone turnover, such as at sites of bone metastasis. It then sequentially delivers a series of four high energy alpha particles. Although the energy of these alpha particles $(3$ to $8 \mathrm{MeV}$ ) is higher than that for various beta particles such as from strontium- 89 or samarium- 153 , the range of the alpha particles is much shorter, being several microns, or less than 10 cell diameters, compared to several $\mathrm{mm}$ for beta particles (Humm, Sartor, Parker, Bruland, \& Macklis, 2015). Ra-223 consequently can kill tumor cells in painful bone metastasis without the same degree of bone marrow toxicity seen with the two FDA approved beta emitters.

The most common non-hematologic adverse effects of Ra-223 experienced by patients in the ALSYMPCA trial included (all grades): bone pain (50\%), nausea (36\%), fatigue $(26 \%)$, diarrhea $(25 \%)$, constipation (18\%), vomiting (18\%), anorexia (17\%), peripheral edema (13\%), and weight loss (12\%) (Parker et al., 2013). Although grades 1 and 2 adverse effects were more common in the Ra-223 patients than in those receiving placebo, grades 3 or higher toxicity were more common among the placebo patients than in those receiving Ra-223. Only $13 \%$ of 
the ALSYMPCA patients receiving Ra-223 had progression of malignancy, and only $4 \%$ had deterioration in their general physical health. This contrasts sharply with our patients, of whom 5/11 (45\%) stopped treatment early due to progression of malignancy or clinical deterioration. The rates of anemia, neutropenia, and thrombocytopenia were no greater in our patients than those receiving Ra-223 in the ALSYMPCA trial.

The ALSYMPCA trial randomly assigned patients to receive best supportive care plus either Ra-223 or placebo delivered every four weeks for six cycles. Patients were stratified as to whether they had previously received docetaxel. Best supportive care at that time consisted of therapies such as bicalutamide, corticosteroids, bisphosphonates, and estrogens. Currently patients have the additional options of abiraterone, cabozantinib, and enzalutamide (Parker, 2015), none of which were available to the ALSYMPCA patients. Our patients received a variety of these agents either prior to or upon completion of Ra-223. Physician preference for the newer agents likely contributed to the small number of patients receiving Ra-223.

Several recent reviews have compared the effectiveness of Ra-223 compared to these newer agents, as well as the beta emitting radiopharmaceuticals Strontium-89 and Samarium-153 (Harrison, Wong, Armstrong, \& George, 2013), (Vignani et al., 2016), (Seal, Asche, Puto, \& Allen, 2013). The cost effectiveness of Ra-223 has also been the subject of a recent report (Norum, Traasdahl, Totth, Nieder, \& Olsen, 2015).

The ALSYMPCA patients had a much longer overall survival than our patients (median survival 14.9 months versus 7.8 months). There are potentially multiple factors that can account for this discrepancy. Our patients were older than those in the ALSYMPCA trial, with a median age of 81, as compared to 71 on the ALSYMPCA trial. The ALSYMPCA trial included 171 patients on the Ra-223 arm and 90 patients on the control arm who were over age 75 , but, to the authors' knowledge, survival and tolerance data on these patients have not been reported separately. It is unclear whether our patients did poorly due to their advanced age or to their prior treatment with multiple lines of therapy.

In this regard, 10 of 11 patients (91\%) received Ra-223 either as last-line (six patient's) or next to last-line (four patients), including 7 of our 11 patients (64\%) who received Ra-223 only after progression on one or more of the newer agents unavailable to the ALSYMPCA patients. This could have had the effect of administering Ra-223 to a greater number of patients with biologically more aggressive and treatment-resistant prostate cancer than in the ALSYMPCA study.

Supporting this concept is the observation that, although it was not statistically significant, the median survival from the first Ra-223 infusion was only 5.3 months for the five patients who received abiraterone prior to Ra-223 compared to 12.65 months for the six who did not ( $\mathrm{p}$-value $=0.247$ ). The estimated difference was -7.35 months with a 95\% CI of $(-19.1,5.0)$. Correspondingly, the recently reported COMET-1 trial found that cabozantinib, an oral inhibitor of tyrosine kinase inhibitors including MET and VEGF receptor-2, did not significantly improve overall survival compared with prednisone in heavily pretreated patients who had bone metastases and disease progression after docetaxel and abiraterone and/or enzalutamide (Smith et al., 2016).

Finally, the factors of advanced age and being pretreated with newer approved agents likely contributed to only $27 \%$ of our patients completing the planned six infusions of Ra-223, as compared to 63\% of the ALSYMPCA patients. In our small group of patients, there was no difference in survival times for those who completed all six planned infusions than for those who received fewer infusions. The authors are unaware of reported survival times from the larger ALSYMPCA trial for those who completed all six infusions compared to those who did not. Of course, our low completion rate may have represented an additional, independent factor in our significantly lower overall survival duration.

Realizing that the survival time of our patients was much less than those reported from the Ra-223 patients in the ALSYMPCA trial, we also compared the survival time of our patients to the control group of the ALSYMPCA trial to determine if our patients had a survival benefit from treatment intermediate between the reported ALSYMPCA control and the ALSYMPCA Ra-223 patients, but our patients' survival was not statistically significant from the ALSYMPCA control group. Since ours was an observational study rather than a controlled clinical trial, we did not have a clearly defined group of patients to serve as an institutional placebo (control) group.

In regards to the measured serum tumor markers, PSA and alkaline phosphatase, the former is not a reliable indicator of tumor response to Ra-223. In the ALSYMPCA trial, 68\% of patients who previously received docetaxel had an increase in their PSA, as did 57\% of men with no prior docetaxel. PSA values before and at the end of treatment were available for nine of our patients, seven (78\%) of whom had a PSA increase at the end of Ra-223. 
In contrast, alkaline phosphatase is considered a much better marker for tumor response. $48 \%$ of the ALSYMPCA patients with prior docetaxel and $46 \%$ of the patients with no prior docetaxel had at least a $30 \%$ reduction in their alkaline phosphatase. Information was available for nine of our patients, four of whom (44\%) had at least a 30\% reduction in their alkaline phosphatase at the end of Ra-223, like the ALSYMPCA patients.

\section{Conclusions}

Not all patients with symptomatic bone-only metastatic castration resistant prostate cancer benefit from Ra-223 to the same extent as the ALSYMPCA patients. This small study is not meant to contest the findings of the large, international ALSYMPCA trial. It is meant only as a caution to community physicians that older patients who have been treated with newer agents such as abiraterone and enzalutamide may find little benefit from the addition of Ra-223. The optimal placement of Ra-223 in the landscape of metastatic castration resistant prostate cancer treatment is unknown and will require the completion of further prospective randomized clinical trials. Until the results of these trials become available, it is prudent to carefully follow the tolerance and survival of Ra-223 patients treated with newer agents such as abiraterone and enzalutamide.

\section{References}

Harrison, M. R., Wong, T. Z., Armstrong, A. J., \& George, D. J. (2013). Radium-223 chloride: a potential new treatment for castration- resistant prostate cancer patients with metastatic bone disease. Cancer Management and Research, 5, 1-14. http://dx.doi.org/10.2147/cmar.s25537.

Hess, K. R., Varadhachary, G. R., Taylor, S. H., Wei, W., Raber, M. N., Lenzi, R., \& Abbruzzese, J. L. (2006). Metastatic patterns in adenocarcinoma. Cancer 106, 1624-1633. http://dx.doi.org/10.1012/cncr.21778.

Hoskin P., Sartor, O., O’Sullivan, J. M., Johannessen, D. C., Helle, S.I., Logue, J., ... Parker, C. (2014). Efficacy and safety of radium-223 dichloride in patients with castration-resistant prostate cancer and symptomatic bone metastases, with or without previous docetaxel use: a prespecified subgroup analysis from the randomized, double-blind, phase 3 ALSYMPCA trial. Lancet Oncol 15, 1397-1406.

http://dx.doi.org/10.1016/S1470-2045(14)70474-7.

Humm, J. L., Sartor, O., Parker, C., Bruland, O. S., \& Macklis, R. (2015). Radium-223 in the treatment of osteoblastic metastasis: a critical clinical review. Int $J$ Radiation Oncol Biol Phys. 91, 898-906. http://dx.doi.org/10.1016/j.ijrobp.2014.12.061.

Koo, K. C., Park, S. U., Kim, K. H., Rha, K. H., Hong, S. J., Yang, S. C., \& Chung, B. H. (2015). Prognostic impacts of metastatic site and pain on progression to castrate resistance and mortality in patients with metastatic prostate cancer. Yonsei Med J. 56, 1206-1212. http://dx.doi.org/10.3349/ymj.2015.56.5.1206.

McDougall, J. A., Bansai, A., Goulart, B. H. L., McCune, J. S., Karnopp, A., Fedorenko, C., .. Ramsey, S. D. (2016). The clinical and economic impacts of skeletal-related events among Medicare enrollees with the prostate cancer metastatic to bone. The Oncologist, 21, 320-326. http://dx.doi.org/10.1634/theoncologist.2015-0327.

Moriera, D. M., Howard, L. E., Sourbeer, K. N., Amarasekara, H. S., Chow, L. C., Cockrell, D. C., ... Freedlan, S. J. (2016). Predictors of time to metastasis in castrate-resistant prostate cancer. Urology, 96, 171-176. http://dx.doi.org/10.1016/j.urology.2016.06.011.

Nørgaard, M., Jensen, A. O., Jacobsen, J. B., Cetin, K., Fryzek, J. P., \& Sørensen, H. T. (2010). Skeletal related events, bone metastasis and survival of prostate cancer: a population based cohort study in Denmark (1999 to 2007). Journal of Urology, 184, 162-167. http://dx.doi.org/10.1016/j.juro.2010.03.034.

Norum, J., Traasdahl, E. R., Totth, A., Nieder, C., \& Olsen, J. A. (2016). Health economics and Radium-223 (Xofigo) in the treatment of metastatic castration-resistant prostate cancer (mCRPC): a case history and a systematic review of the literature. Global Journal of Health Science, 8, 1-9.

http://dx.doi.org/10.5539/gjhs.v8n4p1.

Parker, C., Nilsson, S., Heinrich, D., Helle, S. I., O’Sullivan, J. M., Fosså, S. D., ...Sartor, O. (2013). Alpha emitter Radium-223 and survival in metastatic prostate cancer. N Engl J Med, 369, 213-223.

http://dx.doi.org/10.1056/NEJMoa1213755.

Parker, C. (2015). Use of radium-223 in men with metastatic castration-resistant prostate cancer. Clinical Advances in Hematology and Oncology 13, 723-725. PMID 27058995

Perrault, L., Fradet, V., Lauzon, V., LeLorier, J., Mitchell, D., \& Habib, M. (2015). Burden of illness of bone metastases in prostate cancer patients in Québec, Canada: a population-based analysis. Can Urol Assoc J., 9(910), 307-314. http://dx.doi.org/10.5489/cuaj.2707. 
Seal, B. S., Asche, C. V., Puto, K., \& Allen, P. D. (2013). Efficacy, patient-reported outcomes (PROs), and tolerability of the changing therapeutic landscape in patients with metastatic prostate cancer (MPC): a systematic literature review. Value in Health, 16, 872-890. http://dx.doi.org/10.1016/j.jval.2013.03.1628.

Smith, M., De Bono, J., Sternber, C., Le Moulec, S., Oudard, S., De Giorgi, U., ... Fizazi, K. (2016). Phase III study of cabozantinib in previously treated metastatic castration-resistant prostate cancer: COMET-1. JCO 34, 3005- 3013. http://dx.doi.org/10.1200/jco.2015.65.5597.

Vignani, F. V., Bertaglia, V., Buttigliero, C., Tucci, M., Scagliotti, G. V., \& Di Maio, M. (2016). Skeletal metastasis and impact of anticancer and bone-targeted agents in patients with castration-resistant prostate cancer. Cancer Treatment Reviews, 44, 61-73. http://dx.doi.org/10.1016/j.ctrv.2016.02.002.

\section{Copyrights}

Copyright for this article is retained by the author(s), with first publication rights granted to the journal.

This is an open-access article distributed under the terms and conditions of the Creative Commons Attribution license (http://creativecommons.org/licenses/by/4.0/). 